\title{
Defining argumentation attacks in practice: an experiment in food packaging consumer expectations
}

\author{
Bruno Yun $^{1}$, Rallou Thomopoulos ${ }^{2,1, \star}$, Pierre Bisquert ${ }^{2,1}$, and Madalina Croitoru ${ }^{1}$ \\ 1 INRIA GraphIK, LIRMM (CNRS and Univ. Montpellier), F-34392 Montpellier cedex 5 \\ [bruno.yun, madalina.croitoru] @lirmm.fr \\ 2 INRA, IATE Joint Research Unit, F-34060 Montpellier cedex 1 \\ [rallou.thomopoulos, pierre.bisquert] einra.fr
}

\begin{abstract}
In socio-economic systems, where actors are motivated by different objectives, interests and priorities, it is very difficult to meet all involved party expectations when proposing new solutions. Argumentative approaches have been proposed and demonstrated to be of added value when addressing such decision making problems. In this paper we focus on the following research question: "How to define an attack relation for argumentative decision making in socioeconomic systems?" To address this question we propose three kinds of attacks that could be defined in the context of a precise application (packaging selection) and see how the non computer science experts evaluate, against a given set of decision tasks, each of these attacks.
\end{abstract}

\section{Introduction}

Socio-economic systems involve various actors who interact, while motivated by different objectives, interests and priorities. Food supply chains are examples of such complex systems, involving actors from producers to process and packaging industrials, distributors, recycling industry, etc. Conceiving sustainable food systems implies taking into account various kinds of concerns, including environmental issues (limited energy consumption, reduced GHG emissions, etc.), economic issues (limited costs for consumers, viable firms, source of employment, etc.), social issues (creating social link, ensuring good living conditions, etc.), ethical considerations (pursuing fairness and equity principles), sensorial preferences (appreciated taste and flavor), nutritional issues (contributing in healthy diets), sanitary issues (chemical and microbiological norms), and practical issues (shelf life, ease of use, etc.). As a consequence, these different concerns lead to inconsistent expectations. This raises the difficult question of how to best meet such expectations when proposing new solutions for the future.

Various methods of reasoning under such kind of inconsistency have already been proposed to tackle decision making in food supply chains. The main approaches employed by the state of the art are multi-criteria analysis or argumentation. In the rest of the paper we will focus on argumentation based approaches. This is justified by the fact that multi criteria design encounters a limitation which is particularly hard to address

${ }^{\star}$ corresponding author 
within mathematical frameworks: striking the right balance between problem simplification and real-world complexity [21]. Furthermore, in the context of socio-economic systems decision making [9] the strength of argumentation lies in reasoning traceability, discussion fostering and decision explanation, all leading to a better acceptance of the final decision by all parties $[6,20]$.

An argumentation system consists of a set of arguments and a binary relation on that set, expressing conflicts among arguments. Argumentation has been formalized both in philosophy and in computer science $[14,18]$ and applied to decision making (e.g. [7, $11])$, deductive argumentation and defeasible logic programming $[1,17]$ or for modelling different types of dialogues including negotiation or explanation (e.g. [13, 19,3, 4]). In the reminder of this paper we will focus on using argumentation for decision making. Originally, in order to capture a large class of problems, an argument [10] was seen as abstract entity. This abstraction poses problems when aiming to apply argumentation in practice for decision making. While an argument is intuitively understood as a statement for or against an action, the attack relation is much harder to discern. How is the attack relation obtained? Is it declared, deduced or computed? On which rationale is it defined? How should it be interpreted? Does it have a human-intuitive interpretation or a machine interpretation?

Against this background, in this paper we focus on the following research question: "How to define an attack relation for argumentative decision making in socio-economic systems?" Since the added value of argumentation lies in its principled interaction with the user we evaluate the potential answers to the above research question with the users. More precisely we propose three kinds of attacks that could be defined in the context of a precise application (packaging selection) and see how the non computer science experts evaluate, against a given set of decision tasks, each of these attacks.

The salient point of the paper lies in the fact that it represents, to the best knowledge of the authors, the first study in argumentation research that addresses the problem of attack modelling in practice. This is a significant problem since the structure of the argumentation graph is directly dictated by the attacks. Moreover, such structure plays a fundamental role in how difficult reasoning over the argumentation graph is [12, 24].

The paper is structured as follows. After a recall on modelling choices in Section 2 , the packaging case study is presented in Section 3. The experimental protocol of the paper is proposed in Section 4 and its results discussed in Section 5.

\section{Argumentation primer}

Argumentation is a reasoning model based on the construction and evaluation of conflicting arguments [10]. An argumentation process follows three main steps: (1) constructing arguments and counter-arguments via attacks, (2) evaluating the acceptability of the arguments using semantics defined on the resulting argumentation framework of step (1), and (3) obtaining the justified conclusions based on the set of acceptable arguments of step (2). These notions are formalised below (argumentation framework, acceptability semantics). Please note that for introducing acceptability semantics (in this paper we only considered the preferred semantics) we need to introduce three additional notions i.e. conflict-freeness, defence and admissibility. 
Definition 1. (Dung's argumentation framework). An argumentation framework is a pair $A F=(\mathcal{A}, \mathcal{R})$ where $\mathcal{A}$ is a set of arguments and $\mathcal{R} \subseteq \mathcal{A} \times \mathcal{A}$ is an attack relation. An argument $a$ attacks an argument $a^{\prime}$ if and only if $\left(a, a^{\prime}\right) \in \mathcal{R}$.

Definition 2. (Conflict-free, Defense, Admissibility). Let an argumentation framework $A F=(\mathcal{A}, \mathcal{R})$ and $B \subseteq \mathcal{A}$. Then:

$-B$ is conflict-free if and only if $\nexists a_{i}, a_{j} \in B$ such that $\left(a_{i}, a_{j}\right) \in \mathcal{R}$;

$-B$ defends an argument $a_{i} \in B$ if and only if for each argument $a_{j} \in \mathcal{A}$, if $\left(a_{j}, a_{i}\right) \in$ $\mathcal{R}$, then $\exists a_{k} \in B$ such that $\left(a_{k}, a_{j}\right) \in \mathcal{R}$;

- a conflict-free set $B$ of arguments is admissible if and only if $B$ defends all its elements.

A preferred extension is a maximal (with respect to set inclusion) admissible set of arguments.

In the above framework, an argument is abstract and can be differently instantiated according to various contexts [22]. In general, an argument gives a reason for believing a claim, or for doing an action and it is commonly seen as a set of statements composed of one (or more) premise(s) and a conclusion linked by a logical relation. In decision making, arguments can be intuitively understood as being statements to support, contradict, or explain opinions or decisions [2]. More precisely, in decisional argumentation frameworks [16], the argument definition is provided with additional features: the decision (also referred to as action, option or alternative) and the goal (also referred to as target). In some studies arguments are also associated with specific actors. An application of a decision-oriented argumentation framework to a real-life problem concerning food policy can be found in [8], where a recommendation regarding the provision of whole-grain bread was analyzed a posteriori. In that case, each argument is associated with the action it supports. Based on the above rationale, in this paper, the structure for argument modelling is defined as a tuple composed of a target (a goal), a conclusion inferred from the target choice (i.e. considering / or not this target will imply that the following condition will hold), an aligning of the argument with respect to the target (i.e. if the condition holds in the presence or absence of the target) and a priority rank. Formally:

Definition 3. An argument is a tuple $a=(T, C, A, R)$ where:

$-T$ is the target of the argument;

$-C$ is the conclusion of the argument;

$-A \in\{$ for, against $\}$ is the alignment of the argument (i.e. for or against);

$-R \in \mathbf{N}$ is the rank of the argument.

For any argument $a$, we denote by Target( $a$ ) the target of the argument, Conclu$\operatorname{sion}(a)$ the conclusion of the argument $a$, Alignment $(a)$ the alignment of $a$ and $\operatorname{Rank}(a)$ the rank of the argument. In Table 1 the set of arguments considered in our experimentation is given for illustrative purposes. More details about the use case scenario and the experimentation protocol will be provided in the next section.

In structured argumentation (e.g. logic based argumentation frameworks where arguments are obtained as instantiations over an inconsistent knowledge base) three kinds 


\begin{tabular}{|c|l|l|l|c|}
\hline Name & Target & Conclusion & Alignment & Rank \\
\hline$a 1$ & WC & shock_resistant & for & 14 \\
\hline$a 3$ & WC & taste_preservation & for & 8 \\
\hline$a 5$ & WC & can_smell_product & for & 1 \\
\hline$a 7$ & WC & can_see_product & for & 9 \\
\hline$a 11$ & WC & incites_to_eat & for & 4 \\
\hline$a 14$ & WC & ambiant_preservation & for & 18 \\
\hline$a 15$ & WC & refrigerator_preservation & for & 19 \\
\hline$a 16$ & WC & have_aerations & for & 3 \\
\hline$a 19$ & WC & protect_environment & for & 6 \\
\hline$a 20$ & WC & reusable & for & 13 \\
\hline$a 21$ & WC & harmful_effect & against & 22 \\
\hline$a 2$ & PRL & shock_resistant & for & 15 \\
\hline$a 4$ & PRL & taste_preservation & for & 20 \\
\hline$a 8$ & PRL & can_see_product & for & 5 \\
\hline$a 12$ & PRL & incites_to_eat & for & 16 \\
\hline$a 17$ & PRL & have_aerations & for & 17 \\
\hline$a 9$ & PPF & can_see_product & for & 10 \\
\hline$a 22$ & PPF & NOT_reusable & against & 21 \\
\hline$a 6$ & OPC & can_smell_product & for & 7 \\
\hline$a 10$ & OPC & can_see_product & for & 2 \\
\hline$a 13$ & OPC & incites_to_eat & for & 12 \\
\hline$a 18$ & OPC & have_aerations & for & 11 \\
\hline
\end{tabular}

Table 1: List of arguments of the experimentation use case.

of attacks have been defined: undercut, rebut and undermine. [5]. The intuition of these attacks is either to counter the premise of the opposing argument (the undercut), the conclusion (the rebut) or the logical step that allowed the inference between premise and conclusion (undermine). In abstract argumentation the set of attacks is simply considered as given. A particular aspect to be considered is when the argumentation framework is enhanced with a set of preferences (in this paper the preferences over arguments are cardinal and expressed as ranks). Classically, when preferences are present the attack relation can be modified in order to take into account the induced precedence.

In this paper, since the considered definition of the attack places our work between structured and abstract argumentation (i.e. we impose some structure on the argument given the decision task at hand but do not logically formalise the target and the conclusion), we need to decide how the attack is defined. Following the above intuition, attacking an argument could be achieved by raising doubts about its acceptability and (i) questioning its target, (ii) questioning the alignment with respect to the target, or (iii) using the argument rank. Formally, we consider the following three attack relations $\mathcal{R}_{1}$, $\mathcal{R}_{2}$ and $\mathcal{R}_{3}$ :

Definition 4. Let $a, b \in \mathcal{A}$ be two arguments. We say that:

$-(a, b) \in \mathcal{R}_{1}$ iff Target $(a) \neq$ Target $(b)$;

$-(a, b) \in \mathcal{R}_{2}$ iff Target $(a)=$ Target $(b)$ and Alignment $(a) \neq \operatorname{Alignment}(b)$; 
- Let $\succeq$ be a partial order on arguments, $(a, b) \in \mathcal{R}_{3}$ iff Target $(a) \neq \operatorname{Target}(b)$,
Alignment $(a)=$ Alignment $(b)=$ for and $\operatorname{Rank}(a) \leq \operatorname{Rank}(b)$.

Let us consider again the arguments of Table 1 . We have that $(a 1, a 2) \in \mathcal{R}_{1}$ since Target $(a 1)=W C$ and $\operatorname{Target}(a 2)=P R L$. Furthermore, we have that $(a 1, a 21) \in$ $\mathcal{R}_{2}$ since $\operatorname{Target}(a 1)=\operatorname{Target}(a 21)=W C$ and $\operatorname{Alignment}(a 1)=$ for and Alignment $(a 21)=$ against. Finally, we have that $(a 1, a 2) \in \mathcal{R}_{3}$ since $\operatorname{Rank}(a 1) \leq$ $\operatorname{Rank}(a 2)$.

All of the 3 attacks can be a priori justified from examples as being intuitive, and have been in the literature (see e.g. $[8,23]$ ). Our objective here is to propose an a posteriori and experimental evaluation, which has not been proposed. In this evaluation, we will consider several criteria: (i) adequation to human spontaneous way of reasoning; (ii) human interpretability of the results; (iii) added-value of computarization for the human. Adequation to human spontaneous way of reasoning will be used as the prime criterion to assert intuitiveness.

\section{Case study}

In the framework of the Pack4Fresh agri-food research project supported by the INRA and CIRAD research institutes, a study was launched to design optimized innovative food packagings.

Food packagings play an crucial part in the food market, since they perform multiple functions: marketing appeal, information about the provenance of the products, nutrition facts, food preservation as long as possible associated with food waste reduction, logistic practicality such as the possibility to stack the products for instance. However, food packagings also have harmful effects, in particular on the environment, since it generates waste material and requires energy-intensive manufacturing, etc. At the present time, active research is ongoing to design and develop new-generation, biobased, biodegradable, "intelligent" food packagings. It is mainly focused on technical aspects of packaging, such as properties of the materials used, matter flows through the packaging material, etc. Yet, to be acceptable and usable, these new-generation packagings have to take into account the characteristics expected or appreciated by the users at all levels of the food supply chain (production, transportation, distribution, storage, consumption, etc.). Hence the importance of explicitly identifying these expectations, as exhaustively as possible, starting from cases of (i) existing packagings and (ii) simple systems, excluding in particular labelling information and advanced preservation properties such as modified atmosphere.

Pursuing this objective, in this study it was decided to focus on the case of strawberry packagings, because of the local accessibility of this product and its properties as a perishable product, subject to much wastage by consumers due to bad habits. The following four existing alternatives were considered and the aim of the study was to best identify and analyze the pros and cons of each of them:

- A opened plastic container without lid or plastic film (OPC).

- A opened wooden container without lid or plastic film (WC).

- A plastic container with a rigid lid (PRL). 
- A plastic container with a plastic film (PPF).

In order to determine the viewpoints and needs of the wider class of consumers w.r.t. the four previous packagings, a web survey was developed. The survey was given to a representative (with regard to age and socio-professional category) sample of 840 persons. As an example, the questions asked by the survey were of the form: "Do you think that this packaging has a harmful effect on the strawberries?". The possible answers were "Yes", "No" or "Neutral".

In a first step, a pretreatment of neutral answers was performed. Questions with an important part of neutral answers were eliminated and not exploited at all in the rest of the study. Therefore, we considered the ratio of neutral answers should be less than in the case where answers would have been obtained randomly, i.e. less than 1/3.

Then in a second step we extracted statements from the survey representing the general viewpoint of the consumers w.r.t. the packaging as follows. For a given question, let $n_{1}$ be the number of "Yes" answers and $n_{2}$ the number of "No" answers. If the ratio $n_{1} /\left(n_{1}+n_{2}\right)$ is superior to a given threshold $\alpha$, a positive statement is extracted. Conversely, if the ratio $n_{2} /\left(n_{1}+n_{2}\right)$ is superior to $\alpha$, a negative statement is extracted. Otherwise no statement was extracted. The higher the ratio, the prior the rank of the statement is. Again we set $\alpha$ at $2 / 3$ to ensure the results are statistically different from answers that would have been obtained randomly (i.e. $\alpha \approx 0.5$ ). For instance, for the given assertion of whether "the open wooden containers are recyclable", the number of "Yes" answers of the survey participants is 658, whereas the number of "No" answers is 45 . We have $658 /(658+45)>2 / 3$, thus a positive statement for the open wooden container in the form: "The consumers think that the opened wooden container is recyclable" is extracted. The same question concerning the plastic container with the plastic film provided 165 "Yes" answers and 370 "No" answers. Since we have $370 /(165+370)>2 / 3$, a negative statement against the plastic container with the plastic film, of the form: "The consumers think that the plastic container with the plastic film is not recyclable", is extracted.

These statements were transformed into arguments as shown in Table 1. The different kinds of attacks as defined in the previous section were computed. The resulting three argumentation frameworks (sharing the set of arguments) were the inputs for the experimentation protocol described in the next section.

\section{Experimentation protocol}

The experimentation was composed of 3 groups (A,B and C) of 7, 8 and 10 persons respectively, all experts in agri-food research but not necessarily experts in packaging conception. Each group rigorously followed the experimentation's framework described in Figure 1. The difference in the group sizes is an experimental constraint due to logistic reasons. Although reduced, the experiment size is conform to ratio requirements [15] which recommend a minimum number of observations (here participants) equivalent to the number of entries (here arguments).

These three groups were first shown a textual description of the use-case. Then, they were asked to grade the 4 packagings by giving them a score between 1 and 4 (see Figure 2). In Figure 2, the blue bar above $W C_{A}$ means that in group A, there were 


$\begin{gathered}\text { The partici- } \\ \text { pants are asked } \\ \text { to rank the } \\ \text { packagings }\end{gathered}$
$\begin{gathered}\text { The partici- } \\ \text { pants are asked } \\ \text { to rank the } \\ \text { packagings }\end{gathered}$

Fig. 1: Timeline of the experiment conducted

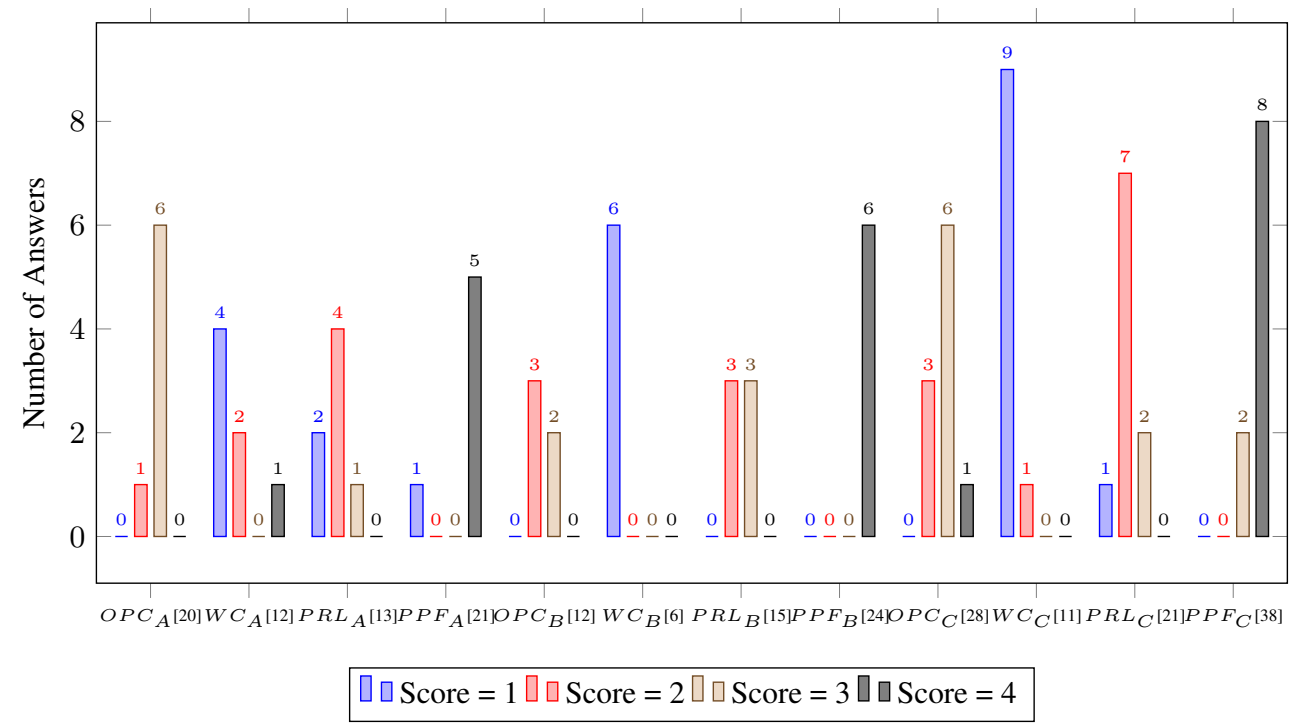

Fig. 2: Scoring for each group before the argumentation methods

4 persons that gave the score 1 to the wooden container whereas the grey bar above $P P F_{C}$ means that in group $\mathrm{C}, 8$ persons gave the score 4 to the plastic container with plastic film. Note that 1 is considered the best score and 4 the worst. The sum may be superior to the number of participants because

The aggregated score of a packaging $p \in\{O P C, W C, P R L, P P F\}$ was computed as $\operatorname{Score}(p)=\sum_{i \in\{1,2,3,4\}}$ NumberPersons $(p, i) * i$.

In the formula above, NumberPersons $(p, i)$ represents the number of persons that gave the score $i$ to the packaging $p$. Please note the smaller the score of a packaging, the better that packaging is. In Figure 2, the aggregated score of each packaging in each group is enclosed in square brackets. The detailed analysis of the results is as follows:

- In group A, the WC is considered the "best" packaging (score 12), the PRL is second (score 13). It is followed by the OPC (score 20) and the PPF at the last place (score 21). 
- In group B, the WC is obviously the "best" packaging (score 6), it is followed by the OPC (score 12) and the PRL (score 15). The all agree to give the PPF the last place (score 24).

- In group C, the WC is the "best" packaging (score 11), the PRL is second (score 21). It is followed by the OPC (score 28) and the PPF is at the last place (score 38).

From the above we can conclude that, before the experiment, the three groups relatively agree on the ranking of packagings: $\mathrm{WC}>\mathrm{PRL}>\mathrm{OPC}>\mathrm{PPF}$ (for groups $\mathrm{A}$ and $\mathrm{C}$ ) and WC $>$ OPC $>$ PRL $>$ PPF (for group B).

The experiment we held out for each group was as follows:

- For group $\mathrm{A}$, we considered the argumentation graph $\mathcal{A S}=(\mathcal{A}, \mathcal{R})$ where $\mathcal{A}$ is the set of arguments of Table 1 with Alignment $(a)=$ for for each $a \in \mathcal{A}$ and $\mathcal{R}=\mathcal{R}_{1}$. They were shown the following list of preferred extensions:

- $\varepsilon_{P P F}=\{a 9\}$

- $\varepsilon_{O P C}=\{a 6, a 10, a 13, a 18\}$

- $\varepsilon_{P R L}=\{a 2, a 4, a 8, a 12, a 17\}$

- $\varepsilon_{W C}=\{a 1, a 3, a 5, a 7, a 11, a 14, a 15, a 16, a 19, a 20\}$

In this case, one preferred extension of the argumentation graph corresponds to one packaging and contains all the arguments that are in favor of this option.

- For group $\mathrm{B}$, we considered the argumentation graph $\mathcal{A S}=(\mathcal{A}, \mathcal{R})$ where $\mathcal{A}$ is the set of arguments of Table 1 and $\mathcal{R}=\mathcal{R}_{2}$. They were shown the following list of preferred extensions:

- $\varepsilon_{1}=\{a 2, a 4, a 6, a 8, a 10, a 12, a 13, a 17, a 18, a 21, a 22\}$

- $\varepsilon_{2}=\{a 2, a 4, a 6, a 8, a 9, a 10, a 12, a 13, a 17, a 18, a 21\}$

- $\varepsilon_{3}=\{a 1, a 2, a 3, a 4, a 5, a 6, a 7, a 8, a 9, a 10, a 11, a 12, a 13, a 14, a 15, a 16, a 17, a 18$, $a 19, a 20\}$

- $\varepsilon_{4}=\{a 1, a 2, a 3, a 4, a 5, a 6, a 7, a 8, a 10, a 11, a 12, a 13, a 14, a 15, a 16, a 17, a 18$, $a 19, a 20, a 22\}$

In this case, one extension cannot contain the arguments for and against one packaging at the same time. Here, $\varepsilon_{1}$ contains the arguments for PRL, the arguments for OPC, the arguments against WC and the arguments against PPF, $\varepsilon_{2}$ contains the arguments for PRL, the arguments for OPC, the arguments for PPF and the arguments against WC, $\varepsilon_{3}$ contains all the arguments for a packaging and $\varepsilon_{4}$ contains the arguments for PRL, the arguments for OPC, the arguments for WC and the arguments against PPF.

- For group $\mathrm{C}$, we considered the argumentation graph $\mathcal{A S}=(\mathcal{A}, \mathcal{R})$ where $\mathcal{A}$ is the set of arguments of Table 1 with Alignment $(a)=$ for for each $a \in \mathcal{A}$ and $\mathcal{R}=\mathcal{R}_{3}$. They were shown the following list of preferred extensions $\varepsilon_{W C}=\{a 1, a 3, a 5, a 7$, $a 11, a 14, a 15, a 16, a 19, a 20\}$, that contains all the arguments for the wooden container.

After conducting the argumentation experiment and showing them the resulting extensions, the participants were asked again to grade the four packagings by giving them a score between 1 and 4 (see Figure 3). Aggregated scores are given in square brackets and the detailed analysis of the results is as followed: 
- In group A, the WC is considered the "best" packaging (score 7), the PRL is second (score 14), third is the OPC (score 21) and the PPF (score 28) is at the last place. Interesting enough, every participant agreed to this ranking.

- In group B, the OPC is the "best" packaging (score 8), it is followed by the PRL (score 10) and WC get the third place (score 16). The PPF is ranked last (score 22).

- In group C, the WC is the "best" packaging (score 8), the OPC is second (score 18). It is followed by the PRL (score 20) and the PPF is at the last place (score 27).

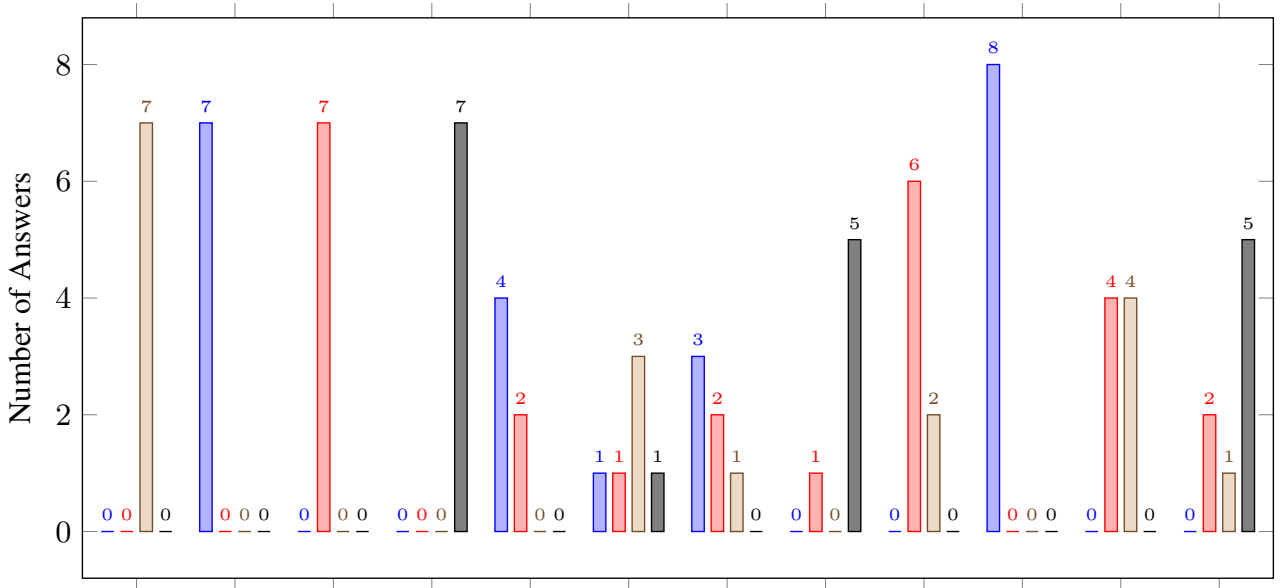

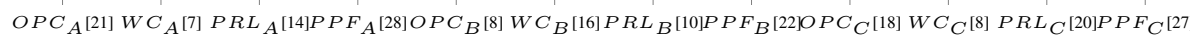

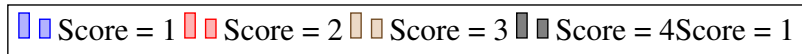

Fig. 3: Scoring for each group after the argumentation methods

Once the scoring of packagings was completed, we reunited the three groups (A,B and C) and proceeded with a group debate. Each group explained its approach and revealed their rankings on packagings. We then asked the three groups to grade again the four packagings by giving them a score between 1 and 4 (see Figure 4). Aggregated scores are given in square brackets and the detailed analysis of the results is as followed:

- In group A, the WC is considered the "best" packaging (score 6), the PRL is second (score 12), third is the OPC (score 18) and the PPF is at the last place (score 24).

- In group B, the WC is the "best" packaging (score 9), it is followed by the OPC (score 19) and PRL at the second/third place (score 19). The PPF is ranked last (score 24).

- In group C, the WC is the "best" packaging (score 10), the PRL is second (score 23). It is followed by the OPC (score 25) and the PPF is at the last place (score 35).

\section{Discussion}

Let us summarise the different rankings expressed in this paper in Table 2. 


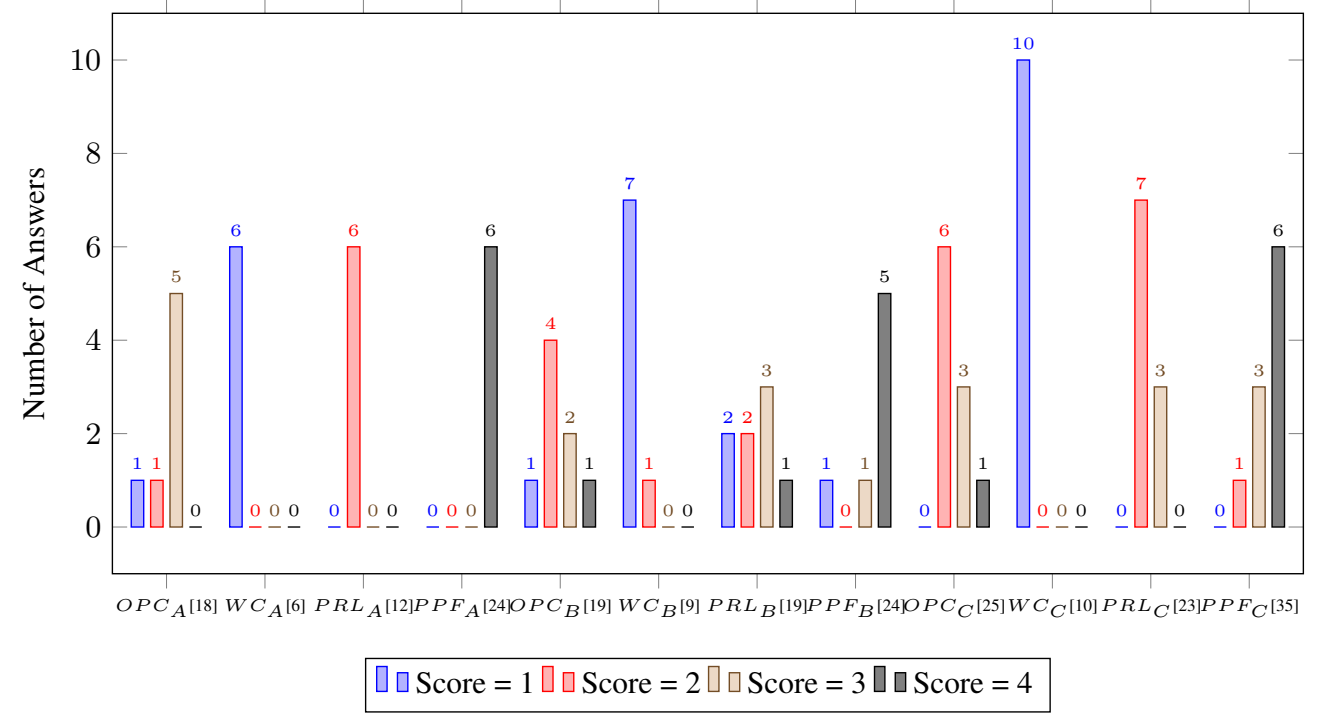

Fig. 4: Scoring for each group after the debate

\begin{tabular}{|c|c|c|c|}
\hline Group & Before the experiment & After experiment & After debate \\
\hline$A$ & $W C>P R L>O P C>P P F$ & $W C>P R L>O P C>P P F$ & $W C>P R L>O P C>P P F$ \\
\hline$B$ & $W C>O P C>P R L>P P F$ & $O P C>P R L>W C>P P F$ & $W C>O P C \sim P R L>P P F$ \\
\hline$C$ & $W C>P R L>O P C>P P F$ & $W C>O P C>P R L>P P F$ & $W C>P R L>O P C>P P F$ \\
\hline
\end{tabular}

Table 2: Overview of the results

From this table we can conclude that the argumentation method of group A does not change the ranking of packagings. The argumentation method of group B seems to be misleading as we can see great changes in the ranking after the experiment which are then revised after the debate. It seems like the argumentation method of group C has some minor effect on the ranking since $P R L>O P C$ before the experiment and $O P C>P R L$ after. One interesting result is that the ranking on packagings is similar before the experiment and after the debate. To go further, let us consider in more detail the ranking statistics as well as the individual comments obtained in each group.

If we examine the statistics of Figures 2 to 4, we can make the following observations. In Figure 2, at the beginning of the experiment before applying the argumentation methods, the global ranking (mixing the answers of the three groups) is clearly $W C>P R L>O P C>P P F$, however the three groups show some nuances in their profiles. Group A shows a clear view about which 2 packagings should be ranked third and last, but seems to be hesitating about how to discriminate between WC and PPF for ranks first and second. Group B shows a clear view of which packaging should be ranked first and last (respectively WC and PPF) but seems more doubtful on the discrimination between the intermediate rankings (PRL or OPC), since the ranking be- 
tween PRL and OPC is close to ex aequo in this group. Group $\mathrm{C}$ offers a quite categoric ranking with little exceptions with $W C>P R L>O P C>P P F$. Since all participants have the same information, the variations observed between the three groups are due to individual differences in interpretation.

In Figure 3, after the experiment applying the different argumentation methods, the differences in the profiles of the three groups become much deeper. Group A provides an answer with no ambiguity with the unanimous ranking $W C>P R L>O P C>P P F$. Thus, this group seems to have gained confidence in their initial answer by applying the argumentation method. Group B, on the contrary, has a much more confused opinion than before the experiment. Indeed, its ranking number one has changed in favour of the packagings that have no negative arguments (OPC and PRL). Its ranking number 2 is now distributed on all the packaging options. WC, which was initially ranked first, has become number 3 because of its negative argument. Only the last ranking, PPF, which also has a negative argument, is unchanged. Group $\mathrm{C}$ has gained confidence in their initial first rank WC, since $100 \%$ of the participants now rank WC first. Their last ranking, PPF, is also confirmed. However ranks 2 and 3 tend to be inversed, with OPC coming before PRL. This can be explained by the prior ranks of the arguments associated with OPC, these ranks being further examined by this group.

In Figure 4, after the debate common to the three groups, the final opinions tend to be more consensual, each group retrieving additional information from the other two experimentations. Hence, the first rank for WC and the last rank for PPF are majoritary in all the groups. Ranks 2 and 3 between OPC and PRL are more controversial, taking into consideration that PRL has more positive arguments, that none of them has negative arguments, but that OPC has prior-ranked arguments.

Moreover, comments given by the participants allow to provide complementary analysis. At the beginning of the experiment before applying the argumentation methods, the comments show that the participants intuitively perform the ranking by seeking a balance between a great number of positive arguments and restricted occurrences of negative arguments, e.g.: "The wooden packaging has lots of positive arguments but also a strong negative argument since it is harmful to strawberries. Thus, the plastic packaging with a rigid lid comes first since it does not have any negative arguments.". "There is a lot of positive arguments and no negative arguments for the plastic container with a rigid lid; The wooden container seems to be ranked second despite of having more positive arguments because of the presence of negative arguments (possibility to smell, harmful effects, etc.)". "I compare the options w.r.t. the number of positive and negative arguments.".

After the experiment, each groups provides interpretations according to the method used. In group A the ranking is reduced to a counting method: "We sort according to the number of arguments. There is a bigger extension for the wooden container.". Group B expresses the difficulty in interpreting the extensions which mix arguments from all packaging options: "There is no possible choice because of the large number of extensions. However, the plastic open container and the plastic container with a rigid lid appear in all the 4 extensions which means that we can prioritise them.". Group C identifies the method is more suitable to compute the best choice than to rank the op- 
tions, since the output contains only one extension here: "The only extension available. The answers for the wooden container are stronger, there is no knowledge on the other packagings".

Based on the observations above, we can conclude on the strong and weak points of each attack definition:

- by separating the positive arguments associated with a given option into a distinguished extension, $\mathcal{R}_{1}$ allows to rank the options by simply counting the number of arguments in each extension, which is what the participants intuitively perform before the experiment. However, $\mathcal{R}_{1}$ does not take into account negative arguments, whereas the participants intuitively do;

$-\mathcal{R}_{2}$ takes into account the bipolar aspect of decision making by considering both positive and negative arguments, as participants intuitively do. But having positive and negative arguments for a given option in separate extensions, and even more mixing arguments from different options in the same extension, is misleading and practically impossible to interprete by the participants;

- the added-value of $\mathcal{R}_{3}$ is to take into account and highlight the prioritization of arguments, which at first glance is not natural to the participants and thus useful to computerize. However the non-symmetric attack relation it provides does not guarantee to keep one extension per option, which is relevant for the selection of best alternatives but leads to a lack of knowledge for the participants about the ranking of the other alternatives.

\section{Conclusion}

In this paper we have introduced an experimental study of a packaging conception use case for strawberries. The aim of the experiment was to investigate how the different attack relations behave on a decision making argumentation framework used by non computer science experts. As seen from our results we can infer that the $\mathcal{R}_{1}$ attack showed to be the most intuitive and easy to use one, whereas $\mathcal{R}_{2}$ was the closest to the bipolar way of reasoning naturally adopted by the participants by considering both positive and negative arguments, and $\mathcal{R}_{3}$ seemed to provide the most added-value in computerizing the approach by performing computations with priority ranks. [23] may be cited as an attempt to combine several of these advantages through a preference-based ranking approach. From the experiment analysed in the present paper, the decision mechanism could be done by an algorithm in order to simulate human experts taking into account attack relations. Obviously from our results, such an algorithm should be primarily based on $\mathcal{R}_{1}$ and possibly refined using $\mathcal{R}_{3}$ then $\mathcal{R}_{2}$.

Let us conclude this paper with one last remark. Further data are available for analysis in the context of this use case as the consumer survey was complemented by interviews with scientists experts of packaging technologies and with actors of the distribution industry (wholesalers, quality managers, etc.). While in this paper we focus on consumers' answers, in order to study the intuitiveness impact of the attack definition in a delimitated case involving only one category of stakeholders, integrating the various stakeholder opinions is ongoing current work. 


\section{Acknowledgements}

The authors acknowledge the participants to the ECIDCM 2016 training school. We also acknowledge the support of the Pack4Fresh project. Many thanks to Patrice Buche for his insights and help with the experimental set up.

\section{References}

1. L. Amgoud, L. Bodenstaff, M. Caminada, P. McBurney, S. Parsons, H. Prakken, J. Veenen, and G. Vreeswijk. Final review and report on formal argumentation system. deliverable d2.6 aspic. Technical report, 2006.

2. L. Amgoud and H. Prade. Using arguments for making and explaining decisions. Artificial Intelligence, 173(3-4):413-436, 2009.

3. A. Arioua and M. Croitoru. Formalizing explanatory dialogues. In International Conference on Scalable Uncertainty Management, pages 282-297. Springer, 2015.

4. A. Arioua and M. Croitoru. A dialectical proof theory for universal acceptance in coherent logic-based argumentation frameworks. In ECAI, pages 55-63, 2016.

5. P. Besnard and A. Hunter. Elements of argumentation, volume 47. MIT press Cambridge, 2008.

6. P. Bisquert, M. Croitoru, F. D. de Saint-Cyr, and A. Hecham. Formalizing cognitive acceptance of arguments: Durum wheat selection interdisciplinary study. Minds and Machines, 27(1):233-252, 2017.

7. B. Bonet and H. Geffner. Arguing for decisions: A qualitative model of decision making. In E. Horvitz and F. Jensen, editors, 12th Conference on Uncertainty in Artificial Intelligence, pages 98-105, Portland, OR, 1996. Morgan Kaufmann.

8. J.-R. Bourguet, R. Thomopoulos, M.-L. Mugnier, and J. Abécassis. An artificial intelligencebased approach to deal with argumentation applied to food quality in a public health policy. Expert Systems with Applications, 40(11):4539-4546, September 2013.

9. B. Delhomme, F. Taillandier, I. Abi-Zeid, R. Thomopoulos, C. Baudrit, and L. Mora. Designing an argumentative decision-aiding tool for urban planning. In OPDE 2017, Montpellier, France, October 2017.

10. P. M. Dung. On the acceptability of arguments and its fundamental role in nonmonotonic reasoning, logic programming and $n$-person games. Artificial Intelligence Journal, 77:321357, 1995.

11. J. Fox and S. K. Das. Safe and sound - artificial intelligence in hazardous applications. MIT Press, 2000.

12. S. A. Gaggl, T. Linsbichler, M. Maratea, and S. Woltran. Benchmark selection at iccma 2017.

13. S. Kraus, K. P. Sycara, and A. Evenchik. Reaching agreements through argumentation: A logical model and implementation. Artif. Intell., 104(1-2):1-69, 1998.

14. J. Mackenzie. Begging the question in non-cumulative systems. Journal of Philosophical Logic, 8:117-133, 1979.

15. A. P. Marugán and F. P. G. Márquez. Decision-Making Management. Academic Press, 2017.

16. W. Ouerdane, N. Maudet, and A. Tsoukias. Argumentation theory and decision aiding, pages 177-208. Springer, 2010.

17. H. Prakken. An abstract framework for argumentation with structured arguments. Argument and Computation, 1(2):93-124, 2011.

18. N. Rescher. The role of rhetoric in rational argumentation. Argumentation, 12(2):315-323, 1997. 
19. K. P. Sycara. Persuasive argumentation in negotiation. Theory and Decision, 28(3):203-242, May 1990.

20. R. Thomopoulos, M. Croitoru, and N. Tamani. Decision support for agri-food chains: A reverse engineering argumentation-based approach. Ecological Informatics, 26(2):182-191, 2015.

21. J. Tremblay and I. Abi-Zeid. Value-based argumentation for policy decision analysis: methodology and an exploratory case study of a hydroelectric project in Québec. Annals of Operations Research, 236(1):233-253, 2016.

22. D. Walton and F. Macagno. A classification system for argumentation schemes. Argument \& Computation, 6(3):219-245, 2015.

23. B. Yun, P. Bisquert, P. Buche, and M. Croitoru. Arguing About End-of-Life of Packagings: Preferences to the Rescue, pages 119-131. Springer International Publishing, Cham, 2016.

24. B. Yun, S. Vesic, M. Croitoru, P. Bisquert, and R. Thomopoulos. A structural benchmark for logical argumentation frameworks. In International Symposium on Intelligent Data Analysis, pages 334-346. Springer, 2017. 